\title{
Ciclo reproductivo y talla media de madurez del congrio colorado, Genypterus chilensis (Guichenot, 1881) en el litoral de Talcahuano, Chile
}

\author{
Reproductive cycle and maturity mean size of the red cusk eel, Genypterus chilensis \\ (Guichenot, 1881) in the coast off Talcahuano, Chile \\ Javier Chong ${ }^{1}$ y Paola González ${ }^{1}$ \\ ${ }^{1}$ Departamento de Ecología Costera, Facultad de Ciencias, Universidad Católica de la Santísima Concepción, \\ Caupolicán 491, casilla 297, Concepción, Chile \\ jvchong@ucsc.cl
}

\begin{abstract}
A study on the reproduction of the red cusk eel Genypterus chilensis was conducted in the fishing area of Talcahuano, Chile. The mean sexual maturity and size at first maturity were determined in a sample of 490 specimens. The microscopic analysis showed the simultaneous presence of oocytes in different maturity stages, in mature individuals. This
\end{abstract}

allows the characterization of the red cusk eel as a partial or multiple spawner. The frequencies of maturity stages in females showed a spawning period between July and October, and a secondary peak in March. The size at first maturity of the red cusk eel was $72.4 \mathrm{~cm}$.

Key words: Reproduction, ovary, histology, mature, Ophidiidae

aportar información sobre el ciclo reproductivo y talla de madurez sexual de G. chilensis.

\section{Material y métodos}

Se analizaron muestras mensuales de congrio colorado, obtenidas de embarcaciones artesanales entre junio 1996 a julio 1997 y que operaron entre caleta Cocholgue (36 32'S) y bahía San Vicente $\left(36^{\circ} 45^{\prime} \mathrm{S}\right)$. En cada ejemplar se registró el largo total $(\mathrm{mm})$, peso total $(\mathrm{g})$ y se determinó el sexo. Se extrajeron las gónadas, que fueron pesadas $(0,1 \mathrm{~g})$, se determinó su grado de madurez macroscópico y se fijaron en formalina neutra al $10 \%$. Para determinar el ciclo reproductivo de G. chilensis, se realizó un examen microscópico de las gónadas y se evaluaron las variaciones del índice gonadosomático (IGS).

El análisis microscópico se realizó en cortes histológicos de $10 \mu \mathrm{m}$ mediante técnicas convencionales. Se preparó el tejido de la sección central del ovario, que fue incluida en histosec y sometida a tinción hematoxilinaeosina. Para la determinación de los estados de madurez ovárica se siguió la escala entregada por Oliva et al. (1986) que reconoce los estados: inactivo, previtelogénico, vitelogénico, maduro, hidratado y en desove (presencia de folículos postovulatorios: FPO).

El índice gonadosomático que indica la tendencia del grado de madurez gonádica en su ciclo reproductivo, se calculó como: 


$$
I G S=\frac{P . \text { gónada }}{P . \text { pez }-P . \text { gónada }} * 100
$$

La talla de primera madurez sexual (TPMS) se estimó de acuerdo al criterio $\mathrm{L}_{50}$, y se utilizó el modelo de Función Logística, considerando como maduro a los ovarios en estado superior a previtelogénico:

$$
P(l)=\frac{1}{1+e^{(\beta 1+\beta 2 * l)}}
$$

donde: $P(l)$ es la proporción de madurez, y $\beta_{1,} \beta_{2}$ son los parámetros de la función logística.

Luego, el cálculo de la talla de la primera madurez deriva de la siguiente expresión que representa la talla a una proporción de madurez dada:

$$
l_{p}=\frac{1}{\beta_{2}} \ln \left(\frac{1}{P}-1\right)-\frac{\beta_{1}}{\beta_{2}}
$$

donde los parámetros $\beta_{1} \beta_{2}$ fueron definidos previamente, $P$ corresponde a la proporción de madurez y $l_{p}$ representa la longitud a una proporción de madurez dada (Hosmer $\&$ Lemeshow 1989). Con la expresión anterior es posible determinar la talla de primera madurez sexual asumiendo que $P=0,5$, así la expresión anterior se simplifica y se reduce a:

$$
l_{50 \%}=-\frac{\beta_{1}}{\beta_{2}}
$$

Finalmente, el intervalo de confianza asintótico es obtenido utilizando el método Bootstrap percentil, a través de la técnica de simulación Monte Carlo incluida en el algoritmo MATSIM (Roa \& Tapia 1998, Roa et al. 1999)

\section{Resultados}

Se analizaron 490 ejemplares de congrio colorado, con un 63\% hembras (LT 40-108 $\mathrm{cm}$ y PT 303-6.300 $\mathrm{g}$ ) y un $37 \%$ machos (LT $41-87 \mathrm{~cm}$ y PT 422 y $2.900 \mathrm{~g}$ ), alcanzando las primeras un rango mayor de tallas.

El examen de los cortes histológicos de ovarios permitió reconocer los estados de madurez microscópicos siguientes: a) E.1 Inactivo, ovarios con ovocitos primarios de forma variada (diámetros hasta $140 \mu \mathrm{m}$ ), núcleo basófilo, grande, esférico, con numerosos nucléolos en su periferia (Fig. 1a); b) E.2 Previtelogénico, ovocitos de mayor tamaño (diámetros 150-240 $\mu \mathrm{m}$ ), con vesículas lipídicas en la periferia del citoplasma, incluye ovocitos primarios (Fig. 1b); c) E.3 Vitelogénico, ovarios con ovocitos grandes (diámetros 250-500 $\mu \mathrm{m}$ ), citoplasma con gránulos vitelo de color oscuro, núcleo grande forma irregular. Se observa la zona radiada y capa granulosa del folículo (Fig. 1c); d) E.4 Maduro, ovocitos con diámetros hasta $650 \mu \mathrm{m}$, citoplasma con gránulos de vitelos muy grandes o densamente teñido por su condensación (Fig. 1d); f) E.5 Hidratado, ovarios con presencia de capas foliculares colapsadas y aplastadas por la salida de los ovocitos al efectuar el corte del folículo (Fig.1e); g) E.6 En desove, ovarios con folículos postovulatorios (FPO) formados por capas (teca y granulosa) colapsadas e invaginadas del folículo, posterior a la expulsión del ovocito hidratado (Fig. 1f). Además, se detectaron folículos atrésicos, folículos en proceso de reabsorción, en todos los estados de los ovarios.

El análisis microscópico mostró ejemplares en estado inactivo todos los meses del año y con mayor frecuencia en enero. El estado de previtelogénesis presentó valores bajos entre agosto y noviembre, para aumentar alcanzando la máxima frecuencia de diciembre a febrero y disminuir de mayo y julio (Tabla 1). Hembras en vitelogénesis se registraron entre agosto y noviembre (1996) y en marzo y julio (1997). Ovarios maduros e hidratados se presentaron entre agosto y octubre de 1996, en marzo y julio de 1997 (Tabla 1), con mayor frecuencia en agosto y julio. Especimenes con FPO se detectaron entre agosto y noviembre de 1996, en marzo y mayo de 1997 con una frecuencia máxima en septiembre (Tabla 1). Folículos atrésicos se registraron entre agosto y diciembre de 1996 con un mayor porcentaje en octubre $(10 \%)$ y diciembre $(8,3 \%)$, mes último que correspondería al término del principal período de desove. De acuerdo con la frecuencia mensual de los EMS, las hembras de G. chilensis presentarían un ciclo reproductivo con un período de madurez y desove principal en invierno-primavera (entre julio y noviembre), seguido de un período de reposo y un desove secundario en marzo, que debería ser corroborado con un muestreo más intensivo.

Los promedios mensuales del IGS mostraron valores muy superiores en las hembras $(0,44-2,25)$ con respecto a los machos $(0,11-0,22)$ evidenciando un hecho poco común, esto es una gran diferencia de desarrollo que experimentan los ovarios con respecto a los testículos en su ciclo de madurez. En hembras, el IGS presentó los valores más altos entre agosto $(1,97)$ y octubre $(1,39)$ y en marzo $(2,25)$, coincidiendo con los períodos de madurez detectados por el examen microscópico de los ovarios; tendencia similar se observó en la variación del IGS en los machos. 
a) E.1 Inactivo

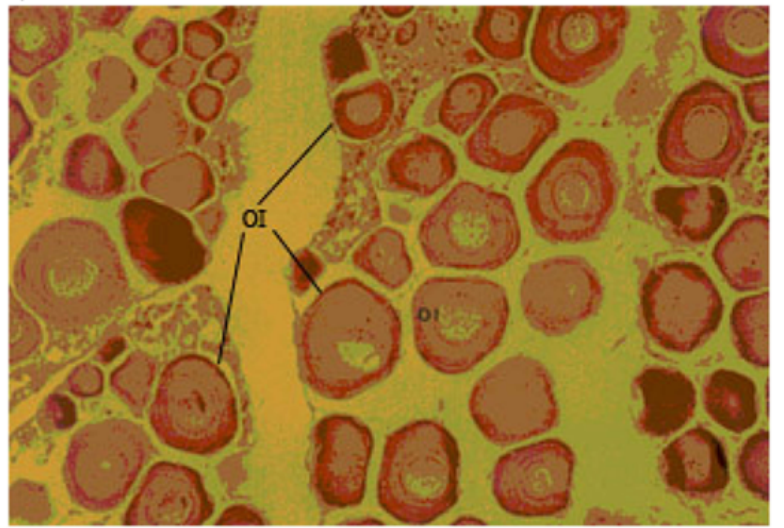

c) E.3 Vitelogénico

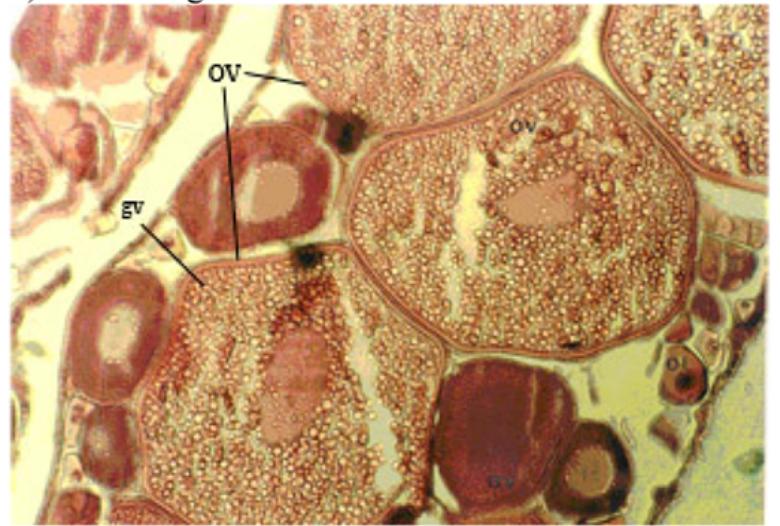

e) E.5 Hidratado

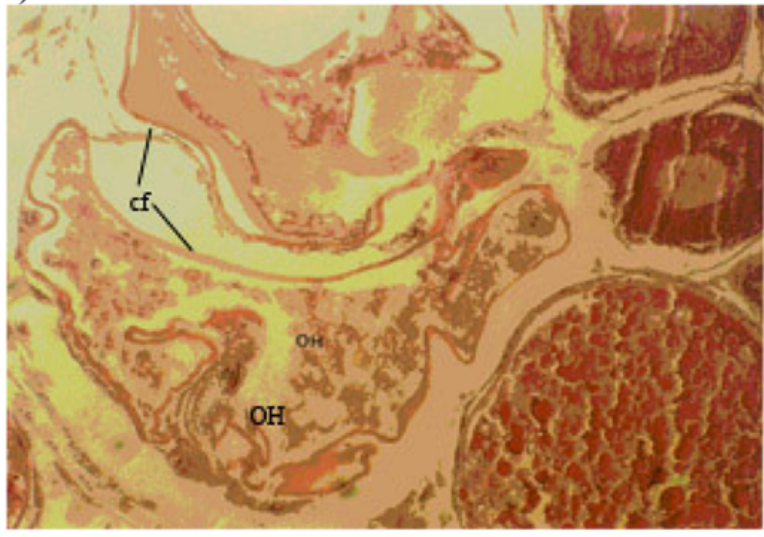

b) E.2 Previtelogénico

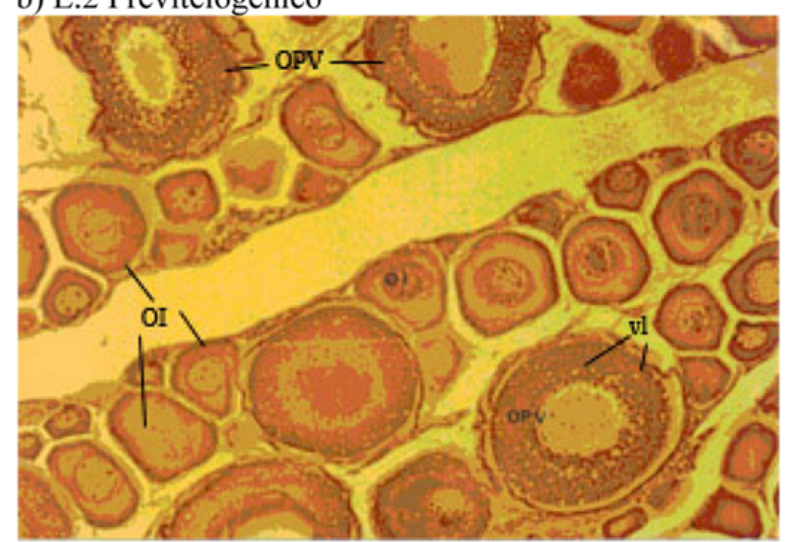

d) E.4 Maduro

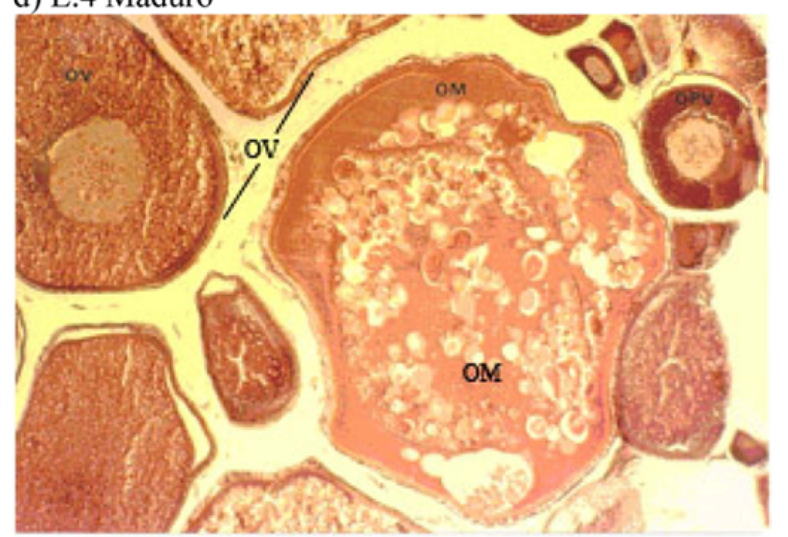

f) E.6 En desove (presencia de FPO)

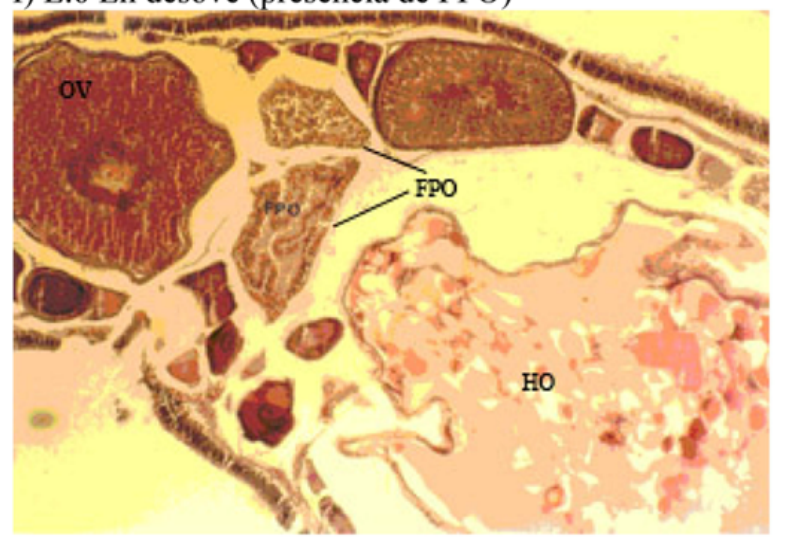

Figura 1

Microfotografías de ovarios de G. chilensis en distintos estados de madurez: a) E.1 Inactivo, se observan sólo ovocitos inactivos (oi); b) E.2 Previtelogénico, ovocitos previtelogénicos (opv) con vesículas lipídicas (vl) en la periferia del citoplasma; c) E.3 Vitelogénico, ovocitos vitelogénicos (ov) con gránulos de vitelo (gv) en el citoplasma; d) E.4 Maduro, ovocitos maduros (om) con citoplasma lleno con gránulos de vitelos, capas folículares (cf) distinguibles; e) E.5 Hidratado, presencia de ovocitos hidratados (oh), capa folicular colapsada y lumen vacío; f) E.6 En desove, presencia de folículos postovulatorios (fpo) (200X)

Photomicrographs of ovaries of G. chilensis in different maturity stages: a) E.1 Inactive, only inactive oocytes (io) are present; b) E.2 Previtellogenic, previtellogenic oocytes (opv) show lipid vesicles (vl) in the outer cytoplasm; c) E.3 Vitellogenic, vitellogenic oocytes

(ov) with yolk granules (gv) in the cytoplasm; d) E.4 Mature, mature oocytes (om) with cytoplasm filled with yolk granules, follicular layer (cf) visible; e) E.5 Hydrated, hydrated oocytes (oh) show a collapsed follicular layer and an empty lumen;

f) E.6 Spawning, ovary with postovulatory follicles (fpo) (200X) 
Tabla 1

\begin{abstract}
Número y frecuencia mensual de estados de madurez microscópicos en hembras de G. chilensis
\end{abstract}
Number and monthly frequency of microscopic maturity stages in females of G. chilensis

\begin{tabular}{|c|c|c|c|c|c|c|c|}
\hline \multirow[b]{2}{*}{ Meses } & \multirow[b]{2}{*}{$\mathrm{N}^{\circ}$} & \multicolumn{6}{|c|}{ Estados de madurez sexual } \\
\hline & & E1 & $\mathrm{E} 2$ & E3 & $\mathrm{E} 4$ & E5 & E6 \\
\hline Junio-96 & 10 & 100 & 0 & 0 & 0 & 0 & 0 \\
\hline Agosto & 25 & 28,6 & 28,6 & 7,1 & 0 & 21,4 & 14,3 \\
\hline Septiembre & 25 & 60,0 & 10,0 & 0 & 0 & 0 & 30,0 \\
\hline Octubre & 90 & 40,0 & 17,1 & 22,9 & 2,9 & 2,9 & 14,3 \\
\hline Noviembre & 45 & 66,6 & 6,7 & 20,0 & 0 & 0 & 6,7 \\
\hline Diciembre & 14 & 50,0 & 50,0 & 0 & 0 & 0 & 0 \\
\hline Enero-97 & 18 & 100 & 0 & 0 & 0 & 0 & 0 \\
\hline Febrero & 26 & 33,3 & 66,7 & 0 & 0 & 0 & 0 \\
\hline Marzo & 30 & 42,1 & 21,1 & 10,5 & 5,3 & 10,5 & 10,5 \\
\hline Mayo & 15 & 70,0 & 10,0 & 0 & 0 & 0 & 20,0 \\
\hline Julio & 11 & 42,9 & 14,3 & 14,3 & 14,3 & 14,3 & 0 \\
\hline
\end{tabular}

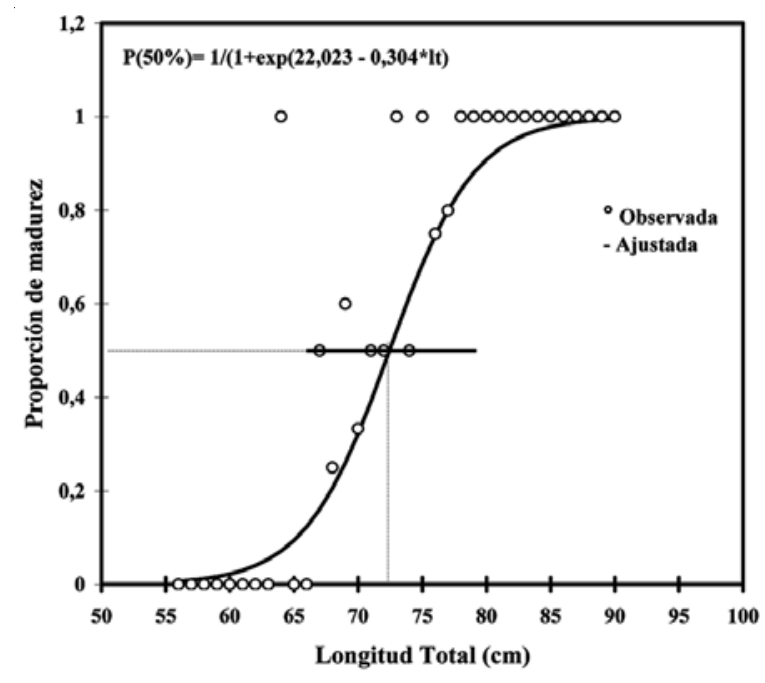

Figura 2

Longitud de primera madurez sexual para congrio colorado estimada mediante función logística, $\left(\mathrm{L}_{50}=72,4 \mathrm{~cm}\right)$

Length of sexual maturity for red cusk eel estimated for logistic function $\left(\mathrm{L}_{50}=72.4 \mathrm{~cm}\right)$

La talla media de madurez sexual se estableció con una muestra de 89 hembras de G. chilensis, en un rango de talla entre 44 y $92 \mathrm{~cm}$ y un rango de IGS entre 0,11 y 9,96 obtenida en la época de máxima madurez sexual (octubre). El modelo logístico entregó un buen ajuste $\left(\mathrm{R}^{2}\right.$ $=0,86)$ con una LT igual a $72,4 \mathrm{~cm}$ para la primera madurez sexual de hembras y los parámetros de la función fueron $\beta_{1}=22,023$ y $\beta_{2}=-0,304$ (Fig. 2).

\section{Discusión}

Los resultados indican que G. chilensis es un desovante múltiple o fraccionado, debido a la presencia de ovocitos en diferentes estados de madurez en un mismo ovario en varios meses del año, lo que se hace más evidente junto a la presencia de FPO en invierno y primavera, que indican desoves recientes (Hunter \& Goldberg 1980, Alarcón et al. 1984). Un tipo de desove similar ha sido registrado por Chong (1976), Tasheri et al. (2003), Paredes \& Bravo (2005) y Andrade \& Matamala (1984), para las especies congenérica G. blacodes (Schneider 1801) y G. maculatus (Tschudi, 1846), respectivamente en la costa de Chile. Esta clase de desove es característico de peces tropicales y temperados y sería una manera eficiente de enfrentar los cambios ambientales, no arriesgando todo el potencial reproductivo en un sólo desove (Nikolsky 1963, Vizziano \& Borois 1990).

El análisis microscópico de ovarios de congrio colorado indica un período de desarrollo gonádico que se inicia en invierno, con un desove máximo entre agosto y octubre y término a fines de la primavera (noviembre); le sigue un período de reposo estival que continúa con un período de madurez, que podría corresponder a un desove secundario a comienzos de otoño (marzo). Un comportamiento reproductivo semejante fue informado por Paz (1991) para G. chilensis del litoral de Coquimbo y por Tascheri et al. (2003) para la costa entre Coquimbo y Lebu, en Chile. En cambio, Paredes \& Bravo (2005) determinan para G. blacodes en la zona sur austral $\left(43^{\circ}\right.$ $56^{\circ} \mathrm{LS}$ ), sólo un período de desove coincidente con el 
desove principal registrado para G. chilensis. Otras especies que habitan en las mismas latitudes presentan un comportamiento gonádico caracterizado por estos dos períodos de puesta, como fue registrado por Balbontín \& Fisher (1981) para Merluccius gayi, Mujica \& Rojas (1984) para Strangomera bentincki y Aguilera et al. (1986) para Sardinops sagax. Lo anterior estaría relacionado con condiciones favorables y alta productividad primaria y secundaria (Arcos \& Wilson 1984, Ahumada et al. 1991) en la zona costera durante este período.

La talla de primera madurez sexual estimada en 72,4 $\mathrm{cm}$ para G. chilensis es superior a la determinada por $\mathrm{Paz}$ (1991) para el litoral de Coquimbo $(64,7 \mathrm{~cm})$; no obstante, esta última es menos confiable porque está basada sobre EMS macroscópicos. Sin embargo, la TPMS es menor a la informada por Tascheri et al. (2003) $(91,87 \mathrm{~cm})$, quienes encontraron una baja incidencia de ejemplares en actividad gonádica, razón por la cual indicaron que su estimación debía ser considerada con reserva. Se carece de información previa sobre este parámetro en $G$. chilensis, como en otras especies congenéricas en Chile, salvo la TPMS media estimada para G. blacodes, de 90 y $84 \mathrm{~cm}$, por Chong (1993) y Paredes \& Bravo (2005), respectivamente, tallas muy superiores a la determinada en el presente trabajo para el congrio colorado.

Llama la atención el alto porcentaje (75\%) de ejemplares bajo la TPMS, tanto en la muestra de este estudio como en el de Tascheri et al. (2003), procedentes de capturas artesanales, lo que indicaría la urgencia de establecer medidas regulatorias para evitar la fuerte explotación de la fracción juvenil de esta especie.

\section{Agradecimientos}

Nuestros agradecimientos a la Dirección de Investigación de la Universidad Católica Santísima Concepción (Proyecto DIN-496) que financió este estudio, así como al Dr. Guillermo Herrera por su crítica revisión del manuscrito. Hacemos extensivos nuestro reconocimiento a dos revisores anónimos cuyos aportes permitieron mejorar el trabajo.

\section{Literatura citada}

Ahumada R, P Matrai \& N Silva. 1991. Phytoplankton biomass distribution and relationship to nutrient enrichment during upwelling event off Concepcion Bay, Chile. Boletín de la Sociedad de Biología de Concepción 62: 1-12.

Arcos D \& R Wilson. 1984. Upwelling and the distribution of Chlorophyll-a within the Bay of Concepción, Chile. Estuarine, Coastal and Shelf Science 18: 25-35.
Aguilera E, C Oyarzún \& J Chong. 1986. Reproductive cycle of the Pacific Sardine, Sardinops sagax musica (Girard, 1854 ) in the fishery area of Talcahuano, Chile (1983-1984). Biología Pesquera 15: 45-53.

Andrade G \& T Matamala. 1984. Microscopía cuantitativa y madurez sexual del congrio negro, Genypterus maculatus (Tschudi, 1846). Seminario de título de Profesor de Estado en Biología y Ciencias Naturales. Departamento de Biología y Tecnología del Mar, Pontificia Universidad Católica de Chile. Talcahuano, $31 \mathrm{pp}$.

Avilés S. 1979. Congrio colorado, Genypterus chilensis (Guichenot) Teleostomi, Perciformes, Ophidiidae. En: IFOP (ed). Estado actual de las principales pesquerías nacionales. Bases para un desarrollo pesquero. Tomo I, Peces 14: 113, CORFO, IFOP, Santiago.

Alarcón VH, SR Goldberg \& J Alheit. 1984. Histología de folículos postovulatorios de la sardina (Sardinops sagax) del Perú. Boletín, Instituto del Mar del Perú 8(1): 1-16.

Balbontín F \& W Fisher. 1981. Ciclo sexual y fecundidad de la merluza, Merluccius gayi gayi, en la costa de Chile. Revista de Biología Marina 17(3): 285-334.

Chirichigno N. 1974. Clave para identificar los peces marinos del Perú. Informe, Instituto del Mar del Perú 46: 1-109.

Chocair J, F Orellana \& J Serra. 1969. Estudio del género Genypterus (congrios) en aguas chilenas (Pisces: Ophidiidae). Tesis de Biología Marina, Universidad de Chile, Valparaíso, 48 pp.

Chong JV. 1976. Algunos aspectos de la biología del congrio dorado Genypterus blacodes (Schneider 1801) de la zona de Talcahuano, Chile. Tesis de Licenciatura en Biología, Universidad de Concepción, Concepción, 112 pp.

Chong JV. 1993. Estimación de fecundidad y talla de primera madurez sexual del congrio dorado (Genypterus blacodes) en la pesquería sur-austral. Informe final. IFOP: 1-24.

Chong, J, K Sepúlveda \& C Ibáñez. 2006. Variación temporal en la dieta del congrio colorado, Genypterus chilensis (Guichenot, 1881) frente al litoral de Talcahuano, Chile (36³2'S-36 45'S). Revista de Biología Marina y Oceanografía 41(2): 195-202.

Hosmer D \& S Lemeshow. 1989. Applied logistic regression, 386 pp. John Wiley and Sons, New York.

Hunter JR \& SR Goldberg. 1980. Spawning incidence and batch fecundity in northern anchovy, Engraulis mordax. Fishery Bulletin 77(3): 641-652.

Mann G. 1954. La vida de los peces en aguas chilenas, 343 pp. Instituto de Investigaciones Veterinarias, Universidad de Chile, Santiago.

Mujica A \& O Rojas. 1984. Fecundidad y estructura poblacional de la sardina común (Clupea bentincki Norman). Investigación Pesquera (Chile) 31: 59-69.

Nikolsky GV. 1963. The ecology of fishes, 352 pp. Academic Press, London. 
Norman JR. 1937. Coast fishes. Part II. The Patagonian Region. Discovery Reports 16: 1-150.

Oliva J, W Carvajal \& A Tresierra. 1986. Biología reproductiva e histología de gónadas en peces, $151 \mathrm{pp}$. Editorial Biocience, Trujillo.

Paredes F \& R Bravo. 2005. Reproductive cycle, size at first maturation and fecundity in the goleen ling, Genypterus blacodes, in Chile. New Zealand Journal of Marine and Freshwater Research 39: 1085-1096.

Paz C. 1991. Dinámica poblacional del recurso congrio colorado Genypterus chilensis (Guichenot, 1948), frente a las costas de Coquimbo. Tesis de Biología Marina, Universidad Católica del Norte, Coquimbo, 76 pp.

Roa R \& F Tapia. 1998. Spatial differences in growth and sexual maturity between branches of a large population of the squat lobster Pleuroncodes monodon. Marine Ecology Progress Series 167: 185-196.

Roa R, B Ernst \& F Tapia. 1999. Estimation of size at sexual maturity: an evaluation of analytical and resampling procedures. Fishery Bulletin 97(3): 570-580.
SERNAPESCA. 2006. Anuario estadístico de pesca 2005, 210 pp. Servicio Nacional de Pesca. Ministerio de Economía, Fomento y Reconstrucción, Valparaíso.

Tascheri R, J Sateler, J Merini, E Díaz, V Ojeda \& M Montecinos. 2003. Estudio biológico-pesquero del congrio colorado, congrio negro y congrio dorado en la zona centrosur. Informe Final FIP 2001-15: 1-300.

Vergara L \& M George-Nascimento. 1982. Contribución al estudio del parasitismo en el congrio colorado, Genypterus chilensis (Guichenot, 1848). Boletín Chileno de Parasitología 37: 9-14.

Vizziano D \& N Berois. 1990. Ciclo histológico del ovario de Macrodon ancylon (Bloch \& Schneider, 1801) (Teleostei: Scienidae). Biología Pesquera 19: 39-47.

Recibido el 21 de noviembre de 2008 y aceptado el 28 de enero de 2009 